\title{
A pulse survey: assessment and management of bone loss in spinal cord injury
}

\author{
Yannis Dionyssiotis $\mathbb{D}^{1} \cdot$ Ruth Marshall ${ }^{2,3} \cdot$ Michael D. Stillman ${ }^{4} \cdot$ Leslie R. Morse ${ }^{5}$ Anastasia-Christina Rapidi $\mathbb{D}^{6}$
}

Received: 22 April 2020 / Revised: 12 November 2020 / Accepted: 13 November 2020

(c) The Author(s), under exclusive licence to International Spinal Cord Society 2020

\begin{abstract}
Study design An online survey

Objectives To describe current clinical practices regarding osteoporosis assessment and management in people with Spinal Cord Injury (SCI) and compare them to recommended diagnostic tests and interventions.

Setting An international collaboration surveying professionals working in SCI Medicine.

Methods Online cross-sectional survey regarding clinical practice trends in the assessment and treatment of osteoporosis in people with SCI. Assessment of whether discrete professional characteristics influenced practices and if those practices were consistent with recommendations from professional organizations.

Results Eighty-two professionals working in SCI Medicine completed the survey. Respondents were equally likely to test for bone loss during the post-acute phase (between 4- and 18-months post injury) and after low impact fracture (41.46\% and $42.68 \%$, respectively) and more likely to test during the chronic phase $(51.22 \%)$. The majority of respondents $(n=56,70 \%)$ assessed bone density with DXA at the hip, and many $(48.78 \%, n=40)$ prescribed simultaneous mobilization, vitamin D and calcium to prevent bone loss in the acute, post-acute and chronic phases. A number of evaluations and interventions were inconsistent with best practice recommendations.

Conclusions Given that reported practices for detection and treatment of osteoporosis in SCI are inconsistent and not datadriven, there is need for dissemination and adoption of existing clinical practice guidelines.
\end{abstract}

Supplementary information The online version of this article (https:// doi.org/10.1038/s41394-020-00361-7) contains supplementary material, which is available to authorized users.

Yannis Dionyssiotis

yannis_dionyssiotis@hotmail.com

1 1st Physical Medicine and Rehabilitation Department, National Rehabilitation Center EKA, Athens, Greece

2 South Australian Spinal Cord Injury Service, Hampstead Rehabilitation Centre, Lightsview, SA, Australia

3 Faculty of Health and Medical Sciences, School of Medicine, University of Adelaide, Adelaide, SA, Australia

4 Department of Rehabilitation Medicine, Sidney Kimmel Medical College of Thomas Jefferson University, Philadelphia, PA, USA

5 Department of Rehabilitation Medicine, University of Minnesota School of Medicine, Minneapolis, MN, USA

6 Physical Medicine and Rehabilitation Department, General Hospital G. Gennimatas, Athens, Greece

\section{Introduction}

Spinal cord injury (SCI) causes severe sublesional bone loss and an increased risk of fractures that is not comparable with osteoporosis caused by other conditions [1]. SCIinduced bone loss is caused by different pathophysiologies that are both primary (postmenopausal, age related) and secondary osteoporosis [2]. Loss of trabecular bone occurs within weeks of SCI while cortical bone loss begins 1 year post injury. Neither biochemical bone markers nor the majority of non-invasive bone densitometry methods can differentiate between loss of cortical and trabecular bone. Hence, even substantial trabecular bone loss can be masked by an unchanged cortical bone mass. Different diagnostic and therapeutic approaches should be incorporated in the early stages post SCI to reveal trabecular bone loss [1, 2].

Dual energy X-ray absorptiometry (DXA) is the "gold standard" for assessing bone in SCI [3]. However, it estimates only areal and not truly volumetric densities or geometrical properties. As a result, bone mineral density (BMD) DXA measurement depends on the size of the body, 
which is important in conditions where the body size may change as an effect of a disease (i.e., SCI) [4].

The World Health Organization (WHO) created an operational definition for postmenopausal and age-related osteoporosis that is based on BMD $Z$ - and $T$-score measurements [5]. For men under the age of 50 and premenopausal women, a $Z$-score of -2.0 is diagnostic of secondary osteoporosis. This definition is frequently used in the medical literature [6], and has been endorsed by the 2019 International Society for Clinical Densitometry (ISCD) Position Statement that address bone mineral testing in SCI. This document addresses SCI-specific risk factors for bone loss and provides clinical guidance regarding timing of osteoporosis screening after SCI and the use of DXAderived BMD measurements to estimate fracture risk and to monitor response to therapy [3]. Both the ISCD Position Statement and the German Speaking Society of Paraplegia (DMGP) Guidelines [7] provide evidence-based recommendations for detection and treatment of osteoporosis in SCI. These documents suggest that initial DXA scanning should be performed when the results are likely to influence patient management and that the interval between tests should be individualized to each patient's clinical status $[3,7]$. However, there remains a lack of clarity in many areas of management of SCI-related bone loss, including the role of the fracture risk assessment tool (FRAX score) to predict the 10-year risk of sustaining a hip or other major osteoporotic fracture and whether SCI-specific $T$ and $Z$-scores would improve fracture risk prediction [8].

The purpose of the current survey was to assess clinical practice patterns regarding osteoporosis assessment and management in an international cohort of SCI professionals and to compare them to those advanced by professional societies. We hypothesized that there would be substantial variation in clinical practices and divergence from evidencebased guidelines.

\section{Methods}

\section{Design}

A 13 question survey (Supplementary Appendix 1) was designed to determine how professionals working in SCI Medicine assess and manage bone loss in SCI. Based on a review of existing literature and the authors' own clinical experiences, an initial version was composed and tested by members of International Spinal Cord Society (ISCoS) and several affiliated organizations.

Once the survey was finalized, it was posted to an internet-based platform (SurveyMonkey Inc, San Mateo, CA, www.surveymonkey.com) and advertised in the ISCoS newsletter and to the authors' colleagues in SCI Medicine.
Questions consisted of single and multiselect multiple choice questions with options for an open response if the answer options did not describe their current practice. Respondents were able to check one or more than one appropriate answer in multiselect multiple answer options.

The survey was open from April to December 2019. No identifying information was collected and consent was implied by subjects' participation. No institutional review or permission was sought or obtained, and none was considered necessary.

\section{Data analysis}

Data from the online platform were downloaded into Microsoft $^{\circledR}$ Office Excel then analyzed. Results were expressed as frequencies (n) and percentages (\%) for categorical data, and correlation between categorical variables was examined using the Chi-square test. All tests are twosided; statistical significance was set at $p<0.05$.

\section{Results}

\section{Demographics}

Eighty-two professionals (all physicians except for one physical therapist) completed the survey. Fifty-two (63.4\%) had trained in Physical and Rehabilitation Medicine, 13 (15.9\%) were spinal cord specialists, and the rest were orthopedic surgeons $(13.4 \%)$, neurologists $(3.7 \%)$, endocrinologists $(1.2 \%)$, radiologist $(1.2 \%)$, or physiotherapist (1.2\%). Respondents came from 25 countries on 4 continents, and a slight majority $(59.0 \%)$ had been in practice for $<20$ years.

\section{Screening practices}

When asked about intervals and initiation of screening for osteoporosis, respondents were equally likely to screen during the post-acute phase and after a low impact fracture (41.5\% and $42.7 \%$, respectively). A majority (51.2\%) reported screening individuals with SCI during the chronic phase, and far fewer during the acute phase (first 3 months of injury-19.5\%) or "only when patients requested it" $(11 \%)$. Just under $10 \%$ of respondents never screen for osteoporosis.

When asked how frequently they screen individuals with SCI for bone loss, the largest group (29.3\%) answered "every 2 years." However, there was wide variability, with $28.1 \%$ screening every year and $15.9 \%$ screening "every 6 months for the first 2 years" of injury. Just over onequarter of respondents entered free-text responses including "only after a low impact fracture," "prior to initiating new weight-bearing," and "when necessary." 
In a set of multiselect questions participants were asked which score ( $T$ - or $Z$-) they use to diagnose osteoporosis in persons with SCI. Eight (10\%) reported using both scores, while $59 \%(n=46)$ reported using $T$-score and $53 \%(n=$ $41)$ reported using $Z$-score, $12 \%(n=9)$ responded "other" and $5 \%(n=4)$ did not answer this question. When asked to select all tests they use to evaluate SCI-related bone loss, $56(70 \%)$ chose DXA of the hip, $46(50 \%)$ DXA of the spine, $14(17 \%)$ whole body DXA, 6 (7.4\%) peripheral quantitative computer tomograph (pQCT) and $4(3.7 \%)$ $\mathrm{BMD}$ at the knee. Free response answers included ulnar pQCT, quantitative ultrasound, and X-rays.

Regarding lab work for monitoring bone loss 15 participants $(18.3 \%)$ answered that they measure calcium, parathyroid hormone $(\mathrm{PTH})$, and $25(\mathrm{OH})$ Vitamin D and six respondents reported measuring serum beta crosslaps.

\section{Prevention and treatment of osteoporosis}

Participants were asked several questions about prevention and treatment of SCI-related bone loss. When queried about interventions they employ to prevent bone loss in the acute and post-acute phases of injury, 40 (48.8\%) reported using mobilization in combination with calcium and vitamin D supplementation. Far fewer respondents used mobilization alone (25.6\%), mobilization in combination with antiosteoporotic medications $(18.3 \%)$, or functional electrical stimulation (FES) or vibration therapy $3.7 \%$ for each). Only three respondents reported not intervening to prevent bone loss.

A separate question inquired how participants attempt to slow bone loss in the setting of concerning blood or urine tests. When laboratory results indicate increased bone loss, 61 respondents $(74.4 \%)$ utilized either vitamin D supplementation of mobilization, $48(58.5 \%)$ offered calcium supplementation, and 27 (32.9\%) prescribed oral bisphosphonates. Others offered intravenous bisphosphonates $(23.2 \%)$, subcutaneous denosumab $(19.5 \%)$ or nothing at all $(4.9 \%)$.

When participants were asked to identify one or more answers in a list of possible answers about treatment approaches for ongoing bone loss, 67 respondents $(82 \%)$ answered that they prescribe vitamin D supplementation, 52 (63\%) calcium, $49(60 \%)$ mobilization, 46 (56\%) bisphosphonates with $18 \% \quad(n=15)$ among them preferring intravenous therapy, 12 (15\%) offered subcutaneous denosumab and 8 (10\%) PTH analogue. Interestingly, 48 participants $(59 \%)$ also answered that they provided consultation regarding lifestyle changes in order to prevent fragility fractures $(n=48)$, and $18(22 \%)$ answered that medication prescription required the occurrence of low trauma fractures. Most responses were prescription of either mobilization or vitamin $\mathrm{D}(n=75,92 \%)$. Twenty-four per cent reported prescribing vitamin $\mathrm{D}$ and mobilization in addition to bisphosphonates.

Finally, respondents were asked about management of bone health after fragility fracture. Many participants reported implementing lifestyle changes $(55 \%)$ to prevent future fractures, and most prescribe medications (82\%). Fifty-three $(65 \%)$ offered bisphosphonates (38 gave it orally and 15 gave it intravenously), 17 (20.7\%) prescribed bisphosphonates in addition to calcium and vitamin $\mathrm{D}$, and $9(11 \%)$ used subcutaneous denosumab.

\section{Discussion}

Here we report clinical practices for osteoporosis management after SCI among an international cohort of $82 \mathrm{SCI}$ professionals. We received responses from 24 countries with $2 / 3$ of those from PM\&R physicians. We identified lack of consistency in the clinical approach to osteoporosis screening, bone density testing protocols, interpretation of bone density results, and interventions to mitigate bone loss in acute or chronic SCI.

We identified these clinical variations despite recently published SCI-specific ISCD position statements regarding the use of DXA to diagnose and monitor osteoporosis in SCI [3]. The position statements recommend DXA scanning at the hip, distal femur, and proximal tibia in all adults with SCI as soon as medically stable after injury. DXA at these sites can be used to "diagnose osteoporosis, predict lower extremity fracture risk, and monitor response to therapy where normal data are available". While there was lack of consensus in the responses regarding use of $T$ - or $Z$-score to diagnose osteoporosis, the ISCD position statements also clarify that $T$ - or $Z$-score should be used based on the individual's age or hormonal status to diagnose SCI-induced osteoporosis consistent with the recommendations for the general population. However, it is acknowledged that fracture risk may be greater for every unit decline in $T$ - or $Z$-score after SCI compared to the general population. Recommendations for testing interval state that, "serial DXA assessment of treatment effectiveness among following a minimum of 12 months of therapy at 1- to 2-yr intervals". Testing should be performed when the results are likely to influence patient management and the interval should be individualized to each patient's clinical status [3]. This is generally 1 year after initiation or change of therapy. The testing interval may be longer in the case of stable therapeutic effects and shorter in cases of accelerated bone loss (such as in acute SCI or with glucocorticoid treatment). More than half of the respondents report using spine DXA to screen for osteoporosis in SCI. However, the ISCD SCI position statements do not recommend DXA testing of the spine after SCI due to inaccurate measurements associated with instrumentation and posterior 
element artifacts. Similarly, regional analyses of whole body DXA measurements are not endorsed by the ISCD. Few respondents report using DXA of the distal femur or proximal tibia to diagnose osteoporosis despite these skeletal sites being the most frequently fractured after SCI. This is likely due to the limited software availability by DXA machine manufacturers to determine bone density at the distal femur and proximal tibia. In the absence of BMD knee measurement software, ISCD recommends the Toronto Rehab Protocol that uses lumbar spine software for determination of BMD [3].

We also report large variations in therapeutic approaches to prevent or treat osteoporosis among respondents. Distinct from the ISCD DXA-based guidelines, there are few established treatment guidelines to direct clinical best practices [7]. Roughly $20 \%$ of respondents endorsed mobilization and antiresorptive drugs to prevent bone loss in acute SCI. A recent point-counterpoint editorial series addressed divergent opinions in osteoporosis prophylaxis, underscoring the need for randomized, placebo-controlled trials to demonstrate the benefit of antiresorptive medication in acute and postacute SCI [9-11]. The field would benefit greatly from evidence-based treatment guidelines for both prophylaxis of bone loss and treatment of osteoporosis after SCI.

There are some limitations of the current study to consider. First, our response rate was low, as is commonly seen with emailed surveys with wide distribution. While we made every effort to distribute this survey as broadly as possible, there are currently no reliable estimates of the number of SCI specialists worldwide and it is unknown how many of those specialists are treating bone loss. Also, while the survey is international in nature, it is not representative of some regions with poor response rates from ASIA and Africa. In addition, the responses are selfreported and we cannot confirm actual prescribing practices. Despite these limitations, we believe the findings shed light on current practices. The responses suggest a lack of standard practices internationally and support ongoing dissemination efforts of recently established clinical guidelines as well as additional clinical trials to determine intervention efficacy.

\section{Compliance with ethical standards}

Conflict of interest The authors declare that they have no conflict of interest.

Publisher's note Springer Nature remains neutral with regard to jurisdictional claims in published maps and institutional affiliations.

\section{References}

1. Jiang SD, Dai LY, Jiang LS. Osteoporosis after spinal cord injury. Osteoporos Int. 2006;17:180-92.

2. Dionyssiotis Y. Spinal cord injury-related bone impairment and fractures: an update on epidemiology and physiopathological mechanisms. J Musculoskelet Neuronal Interact. 2011;11: 257-65.

3. Morse LR, Biering-Soerensen F, Carbone LD, Cervinka T, Cirnigliaro CM, Johnston TE, et al. Bone Mineral Density Testing in Spinal Cord Injury: 2019 ISCD Official Position. J Clin Densitom. 2019;22:554-66.

4. Willnecker J. Measurement of bone density and bone strength with p QCT. In: Weinbauer GF, Vogel F (eds). Novel approaches towards primate toxicology. (Waxmann, 2006), pp. 13-19.

5. World Health Organisation. Assessment of fracture risk and its implication to screening for postmenopausal osteoporosis: technical report series 843. Geneva: WHO; 1994.

6. Sambrook P, Schrieber L, Taylor T, Ellis A. The musculoskeletal system. Edinburgh: Churchill Livingstone; 2001.

7. Frotzler A, Moosburger J, Kalke YB. Spinal Cord Injury related osteoporosis: The draft of the working group for Osteoprosis of the German-speaking Medical Society of Paraplegia (DMGP) as a basis for discussion. Osteol/Osteol. 2015;24:85-91.

8. Lewiecki EM, Compston JE, Miller PD, Adachi JD, Adams JE, Leslie WD, et al. FRAX $\left({ }^{\oplus}\right)$ Bone Mineral Density Task Force of the 2010 Joint International Society for Clinical Densitometry \& International Osteoporosis Foundation Position Development Conference. J Clin Densitom. 2011;14:223-5.

9. Morse L. Osteoporosis prophylaxis in acute SCI. Spinal Cord Ser Cases. 2019;5:27.

10. Dionyssiotis Y. Is prophylaxis for osteoporosis indicated after acute spinal cord injury? Spinal Cord Ser Cases. 2019;5:24.

11. Anderson D, Park AJ. Prophylactic treatment of osteoporosis after SCI: promising research, but not yet indicated. Spinal Cord Ser Cases. 2019;27:25. 